\title{
Investigating the Relationship between Coverage Area and Propagation Profile
}

\author{
Adenodi Raphael Adewale \\ Physics Department, Adeyemi College of Education, Ondo, Nigeria
}

Received: 24 November 2018; Accepted: 16 February 2019; Published: 08 March 2019

\begin{abstract}
This study investigates the relationship between coverage area and propagation profile. Most studies on propagation profile do not link the profile with the coverage area of the transmitter and vice versa. Using both coverage area and propagation profile to describe the variations of electric field intensity of propagating wave makes a vivid description of the transmitter performance. This study creates propagation profile from the contour of coverage area of Benue Radio transmitter. Data for the study were collected during a measurement campaign carried out around the transmitter. The data were interpolated to produce a contour of the coverage area. A cross sectional profile elicits from the contour of coverage area at equally spaced eight different vertical plains provides the propagation profiles of the transmitter. This was achieved by transforming the coordinates of the contour of coverage area into distance thus producing a two dimensional plain from a three dimensional contour. Values of coordinates and electric field intensity were taken from some points on the contour and were compared with the equivalent positions on the propagation profile yields the same value of electric field intensity and equivalent position in distance. Similar procedure was carried out on the propagation profile provides the same value of electric field intensity and equivalent positions in terms of coordinates. The study concludes that propagation profiles are cross sectional parts of coverage area.
\end{abstract}

Index Terms: Transmitter, coverage area, propagation profile, contour, electric field intensity.

(C) 2019 Published by MECS Publisher. Selection and/or peer review under responsibility of the Research Association of Modern Education and Computer Science

\footnotetext{
* Corresponding author. Tel.:

E-mail address: adenodiraph@gmail.com
} 


\section{Introduction}

The performance of a radio transmitter is described in terms of the behavior of the electric field intensity of the radio wave generated by the transmitter. This behavior includes but not limited to the variations of the electric field intensity as the wave propagate, and this will finally determined its propagation profile and coverage area. Both are closely connected but are used in different ways to described variations in the signal and the extent of its coverage. Propagation profile is a two dimensional plot of electric field intensity and distance [1] while coverage area is a three dimensional plot of electric field intensity and coordinates of points measurements [2]. Their vertical and horizontal axes represent respectively the signal strength and the distance covered. Propagation profile traces signal along a route or a vertical plane within the coverage area. Coverage area practically covers the landmass the transmitter is licensed to serve and therefore a lot of propagation profiles will be required for a description equivalent to that of coverage area. When the need to fully understand the behavior of the signal along vertical plain arises, propagation profile is most preferable. Each has its distinctive usefulness and plays complementary roles in a vivid understanding of the performance of radio transmitter. This study investigates the relationship and complementary roles of propagation profile and coverage area in describing the performance of radio performance of transmitter of radio broadcasting station.

According to [3], the competitions between broadcasters to win the interest of the populace necessitate the need to conscientiously measure both the coverage area and the signal quality. A very large coverage area reaching many localities with quality signal and clear reception will provide increased patronage [4]. If the coverage area is large but the quality of the signal is poor in some regions, propagation profile of the signal will be needed to ascertain degree of decay of the electric field intensity along the propagation paths. Reason for a sudden decline in the electric field intensity, such as blockage of radio path by building, can be detected if the location is known [5]. Apart from the good quality and developmental needs provided by the program contents, both coverage area and propagation profile are essentially useful for marketability of broadcasting stations. The higher the values of electric field strength and distance plotted respectively on vertical and horizontal axes, the more the signal fidelity and clarity as well as the propagation distance. This implies wide coverage area with clear signals for the benefit of the listeners.

Most authors used either coverage area or propagation profile to explain the variability of electric field strength around radio transmitter. Coverage areas were employed to study the variability of electric field strength of FM radio [6], VHF/UHF television [7] transmitters in Niger State and MW radio transmitter in Kaduna State [8]. On the other hand, propagation profiles were utilized to describe the variation in the signal strength of UHF [1] in Ondo State and VHF [9] in Ekiti State.

\section{Material and Methods}

According to [10], electric field intensity measurement along a route with coordinates registration by mobile means preferably portable field intensity meter and handheld Global Position System receiver is the most cost-effective means for establishing a very large coverage area. This method was used to collect data of electric field intensity and coordinates along seven routes around Radio Benue transmitter. Field intensity meter was used to measure electric field intensity and Garmin 38 Global Position System receiver records the coordinates of points of measurement. The parameters of transmitter are presented in Table 1. GIS software such as arcgis and surfer are commonly used to produce elevation profiles from digital elevation model [11]. This is done by transforming the coordinates into distance while the vertical axis retains the electric field intensity. The data collected were interpolated in surfer to produce contour of coverage area. The contour was thereafter sliced into eight propagation profiles at equally spaced vertical plains of North, East, South, West, $45^{\circ}$ West of North, $45^{\circ}$ East of North, $45^{\circ}$ West of South and $45^{\circ}$ East of South. The profiling began at the centre of the contour, which is considered to be transmitter location to eight equally spaced locations at the boundary of the contour. It transforms the coordinates of the contour of coverage area into distance thus producing a two dimensional plain from a three dimensional contour [12]. The coordinates of some points on 
the coverage area were selected and the distance between each of these points and the coordinates of the transmitter were calculated to get their equivalent distances on the propagation profiles. This was done to ascertain the utility maintains the varying positions of electric field intensity when converting from coverage area to propagation profile.

Table 1. Parameters of Radio Benue Transmitter

\begin{tabular}{|l|l|}
\hline Transmitter Power & $50 \mathrm{~kW}$ \\
\hline Frequency & $918 \mathrm{kHz}$ \\
\hline Height of Mast & $163.0 \mathrm{~m}$ \\
\hline Coordinates of Mast & $\begin{array}{l}\text { Latitude } 7^{\circ} 41^{\prime} 57.10^{\prime \prime} \mathrm{N} \\
\text { Longitude } 8^{\circ} 32^{\prime} 38.00^{\prime \prime} \mathrm{E} \\
\text { Altitude } 1140.0 \mathrm{~m}\end{array}$ \\
\hline Name of Location & Makurdi, Makurdi LGA, Benue State \\
\hline
\end{tabular}

\section{Result and Discussion}

The coverage areas plotted for the transmitter is presented in Figures 1 and 2 with a minimum electric field intensity of $0.3 \mathrm{mV} / \mathrm{m}$ and an interval of $2.0 \mathrm{mV} / \mathrm{m}$ between any two consecutive levels. The profiling was carried out on Figure 1 but was not possible with the wireframe presented in Figure 2. This is a three dimensional contour having the coordinates as the first two dimensions and electric field level as third the dimension. The electric field intensity is highest at the transmitter location. The data that can be elicited from coverage area are presented in Table 2. All the propagation profiles show that the electric field intensity is high close to the transmitter and smoothly reduces as distance increases. This is in agreement with the wireframe coverage area presented in Figure 2. Table 3 presents the values of the electric field intensity at some distances on the propagation profiles thereby revealing the rate of reduction along the sections. These are not obvious on the coverage areas presented in Figures 1 and 2. The propagation profile along Northern, Southern, Eastern, Western, East of Northern, West of Northern, East of Southern and West of Southern directions are presented in Figures 3, 4, 5, 6, 7, 8, 9 and 10 respectively.

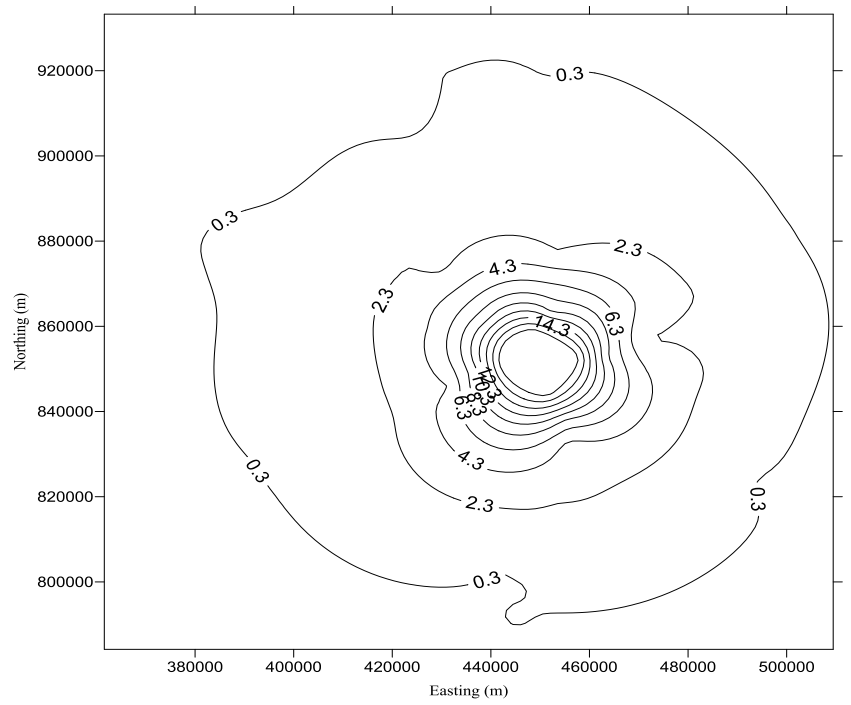

Fig.1. Contour of Coverage Area of Radio Benue 


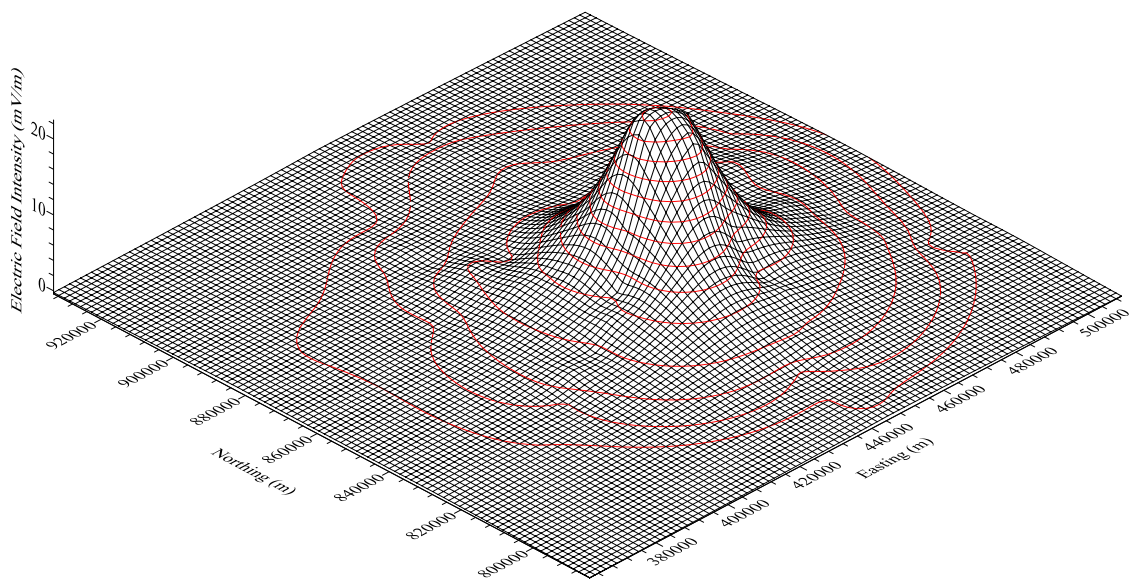

Fig.2. Wireframe of Coverage Area of Radio Benue Transmitter

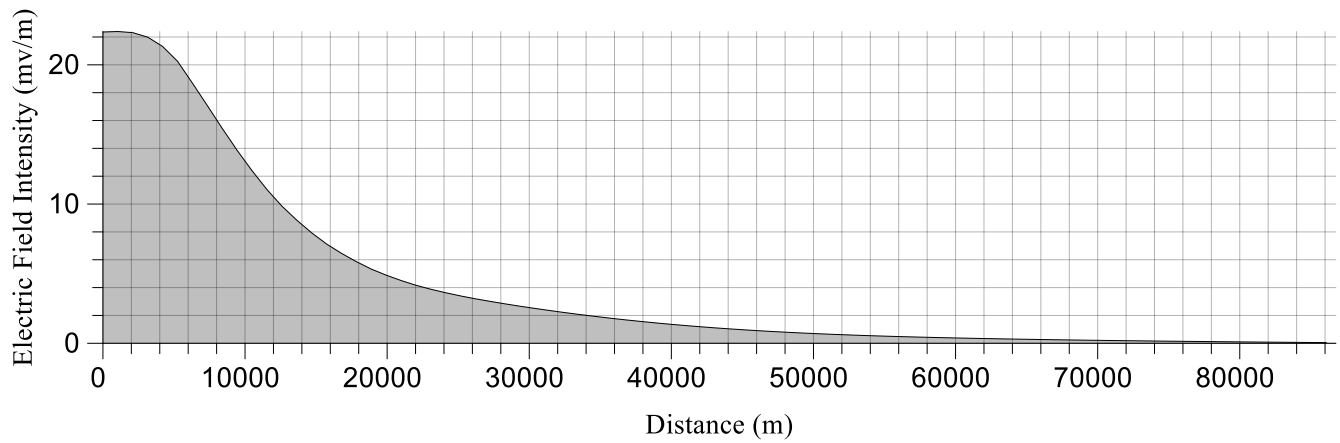

Fig.3. Propagation Profile along Northern Direction

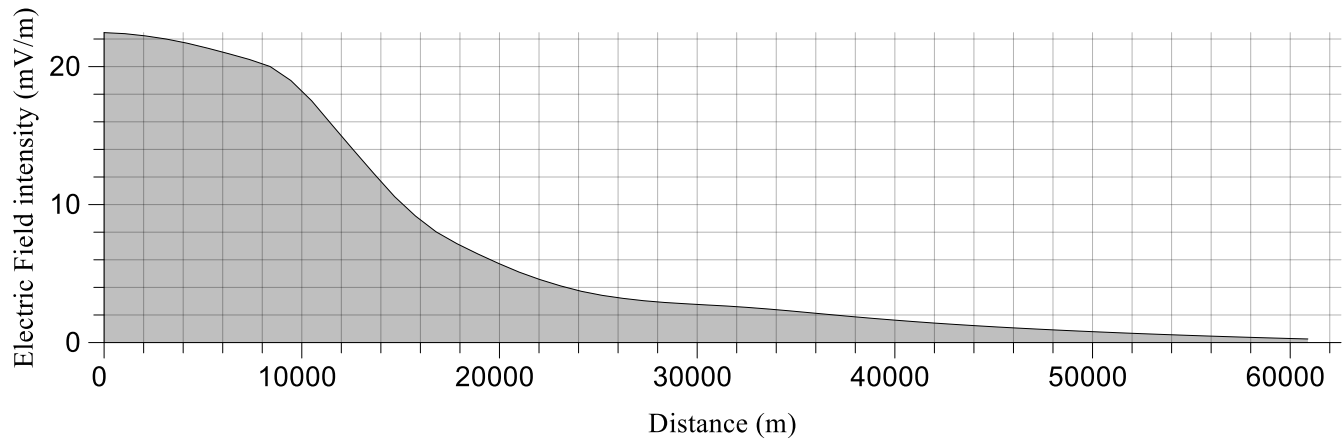

Fig.4. Propagation Profile along Southern Direction 


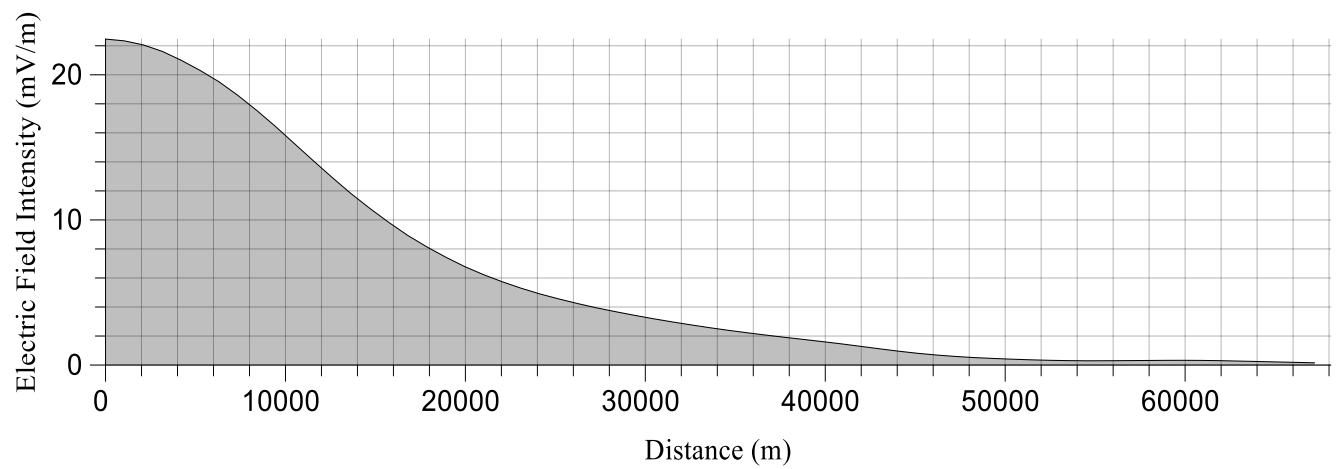

Fig.5. Propagation Profile along Eastern Direction

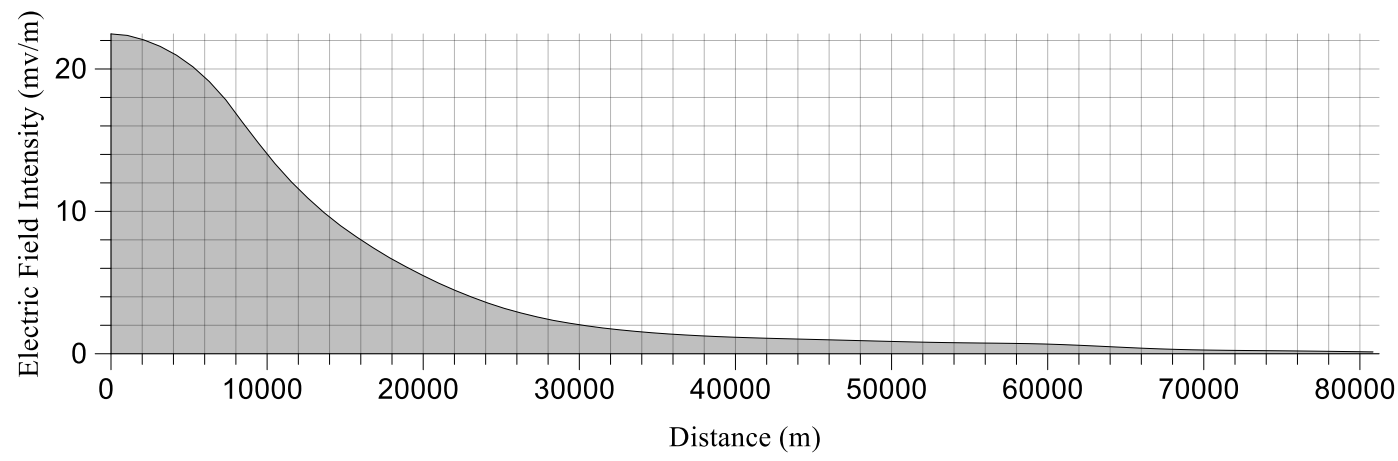

Fig.6. Propagation Profile along Western Direction

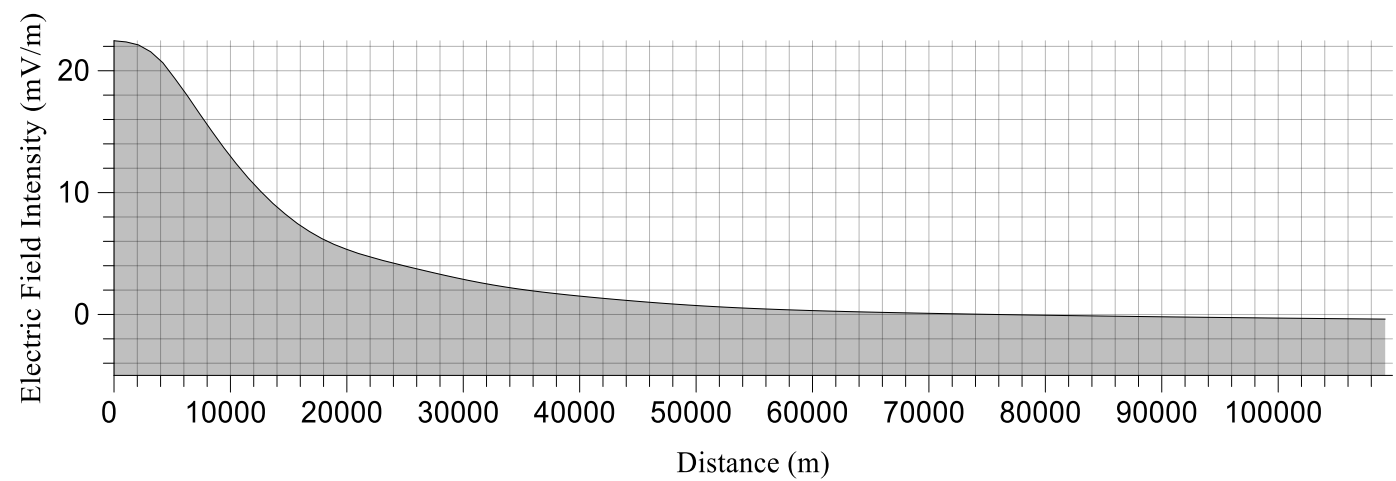

Fig.7. Propagation Profile along $45^{\circ}$ East of Northern Direction 


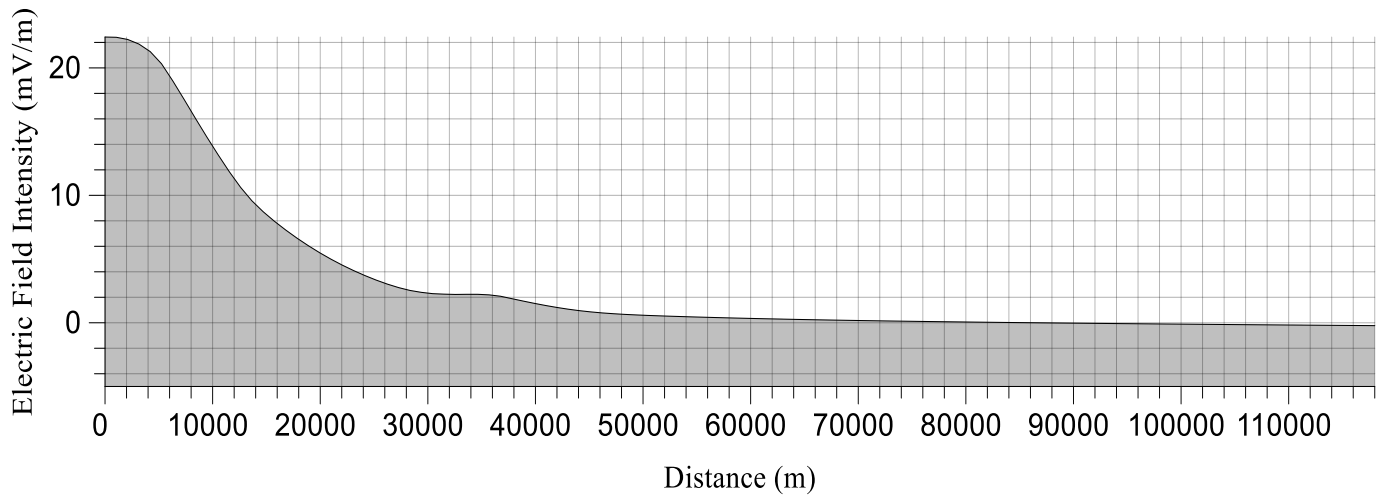

Fig.8. Propagation Profile along $45^{\circ}$ West of Northern Direction

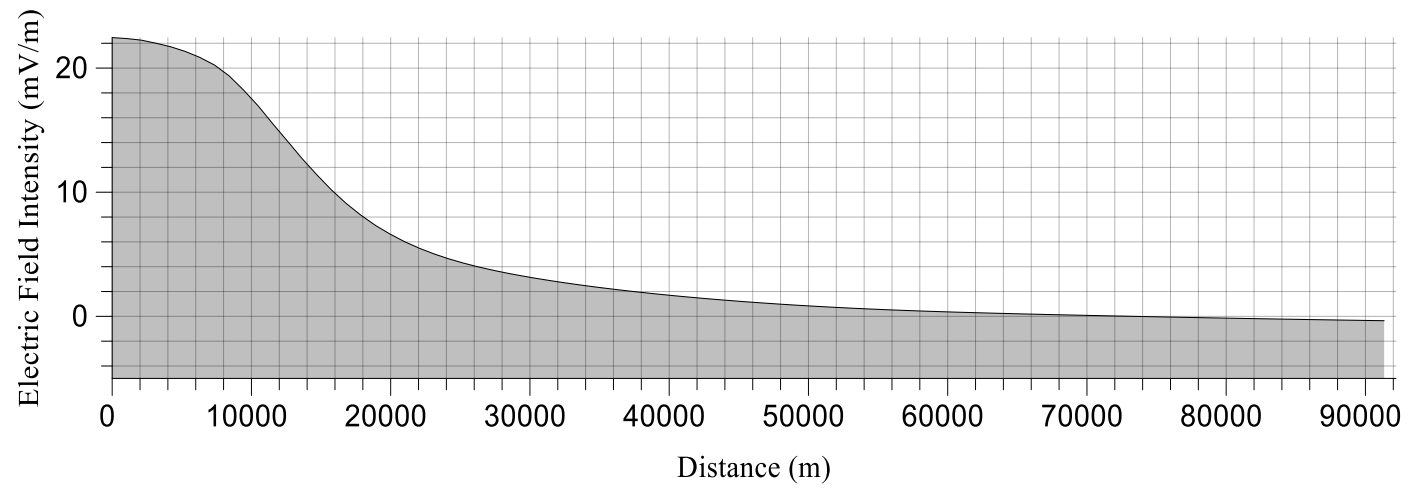

Fig.9. Propagation Profile along $45^{\circ}$ East of Southern Direction

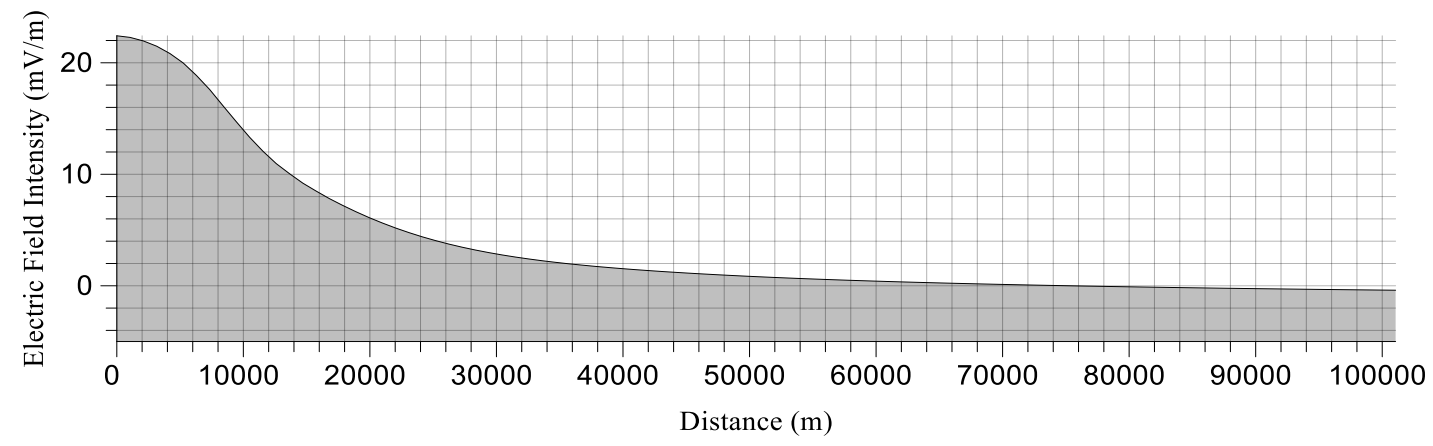

Fig.10. Propagation Profile along $45^{\circ}$ West of Southern Direction

The selected coordinates on the coverage area were found to have the same values of electric field intensity with their equivalent distances on propagation profiles. These are presented in Table 4 . The location of electric field which is expected to be high but flatten out can be traced. The positions of the flatten outs which are conspicuous on the propagation profile can be traced on the coverage area. This is done by transforming the distance on the propagation profile to coordinates on the coverage area as presented in Table 4. Coverage area 
presents the total landmass covered by the transmitter while the propagation profile reveals the condition of the signal strength along selected radial. Their roles are distinct and complementary.

Table 2. Electric filed Intensity at Different Coordinate Points on Coverage Area

\begin{tabular}{|c|c|c|c|}
\hline $\mathrm{S} / \mathrm{N}$ & Latitude $(N, m)$ & Longitude $(E, m)$ & Electric Filed Intensity $(\mathrm{mV} / \mathrm{m})$ \\
\hline 1 & 920000 & 460000 & 0.30 \\
\hline 2 & 880000 & 380000 & 0.30 \\
\hline 3 & 840000 & 420000 & 2.30 \\
\hline 4 & 840000 & 460000 & 8.30 \\
\hline 5 & 860000 & 440000 & 14.30 \\
\hline
\end{tabular}

Table 3. Electric filed Intensity at Different Distances on Propagation Profiles

\begin{tabular}{|c|c|c|c|c|c|c|c|}
\hline \multirow[t]{2}{*}{ Figure } & \multirow[t]{2}{*}{ Direction } & \multicolumn{5}{|c|}{ Values of electric field intensity $(\mathrm{mV} / \mathrm{m})$} & \multirow{2}{*}{$\begin{array}{c}\text { Final Distance } \\
(\mathrm{m})\end{array}$} \\
\hline & & $20,000 \mathrm{~m}$ & $40,000 \mathrm{~m}$ & $60,000 \mathrm{~m}$ & $80,000 \mathrm{~m}$ & $10,000 \mathrm{~m}$ & \\
\hline 3 & North & 7.00 & 1.50 & 0.70 & 0.30 & 0.10 & 80,000 \\
\hline 4 & South & 5.80 & 1.70 & 0.20 & - & - & 60,000 \\
\hline 5 & East & 7.00 & 3.10 & 1.80 & 0.50 & 0.20 & 60,000 \\
\hline 6 & West & 5.80 & 1.00 & 0.40 & 0.10 & - & 80,000 \\
\hline 7 & East of North & 6.00 & 1.80 & 0.30 & 0.10 & - & 90,000 \\
\hline 8 & West of North & 5.80 & 1.80 & 0.60 & 0.10 & - & 90,000 \\
\hline 9 & East of South & 8.00 & 1.90 & 0.40 & 0.05 & - & 80,000 \\
\hline 10 & West of South & 6.00 & 1.80 & 0.40 & 0.05 & - & 80,000 \\
\hline
\end{tabular}

Table 4. Electric Field Intensity at Equivalent Position on Coverage Area and Propagation Profile

\begin{tabular}{|c|c|c|c|c|}
\hline S/N & Latitude $(N, m)$ & Longitude $(E, m)$ & Electric Filed Intensity $(\mathrm{mV} / \mathrm{m})$ & Distance $(\mathrm{m})$ \\
\hline 1 & 824949.930 & 456853.493 & 3.42 & 27.15 \\
\hline 2 & 824029.410 & 387309.686 & 0.24 & 67.98 \\
\hline 3 & 879614.844 & 369125.421 & 0.14 & 85.49 \\
\hline 4 & 919146.32 & 430121.558 & 0.70 & 51.29 \\
\hline 5 & 906269.938 & 453832.681 & 2.10 & 39.73 \\
\hline 6 & 868191.027 & 485494.264 & 2.98 & 30.39 \\
\hline 7 & 848826.892 & 480032.164 & & \\
\hline
\end{tabular}

\section{Conclusions}

The coverage areas are presented in Figures 1 and 2 while the propagation profiles are presented in Figures 3 to 10 . The data that can be extracted from the coverage area are complementary to those from the propagation profiles. Each play unique role in presenting the performance of radio transmitter. The study concludes that propagation profile is an integral cross sectional part of coverage area and both play complementary roles in vivid description of transmitter performance. 


\section{References}

[1] Akinbolatia A., Akinsanmi O. and Ekundayo K. R. "Signal Strength Variation and Propagation Profiles of UHF Radio Wave Channel in Ondo State, Nigeria." I. J. Wireless and Microwave Technologies (IJWMT) DOI: 10.5815/ijwmt.2016.04.02., 4, 12-28 (2016).

[2] Gumusay M. U., Sen A., Bulucu U., and Kavas A. "Electromagnetic Coverage Calculation in GIS. International Symposium on Mobile Mapping Technology”, Padova. Italy. (2007).

[3] Ajewole M. O., Akinbolati A., Adediji A. T. and Ojo J. S. "Precipitation Effect on the Coverage Areas of Terrestrial UHF Television Stations in Ondo State, Nigeria." International Journal of Engineering and Technology 4(9); 524 - 535 (2014).

[4] Ashwin A S., Factor Analysis: "A Study of Consumer Behavior towards FM Radio in Surat City."International Journal of Applied Research 1(12); 1011-1016, (2015).

[5] Holloway C. L., Sanders F. H. and McKenna P. M. "Predicted and Measured Field Strengths in the Boulder, Colorado, Area from Two Proposed Terrestrial Digital Television Tower Sites" NIST Technical Note 1519 and NTIA Report 01-387, 2001

[6] Ajewole M. O., Oyedum O. D., Adediji A. T., Eichie J. O. and Moses A. S. "Spatial Coverage of FM Radio Transmitters in Niger State, Nigeria." The IUP Journal of Telecommunications, IV (4): 7 - 20, (2012).

[7] Ajewole M. O., Oyedum O. D., Adediji A. T., Moses A. S. and Eichie J. O. "Spatial Variability of VHF/UHF Electric Field Strength in Niger State, Nigeria." International Journal of Digital Information and Wireless Communications 3 (3): 26 - 34, (2013).

[8] Adenodi R. A., Ajewole M. O., Ogolo, E. O and Adediji, A. T. "Application of Amplitude Modulated Medium Wave Transmitters in Deploying Advanced Traveller Information System" Australian Journal of Basic and Applied Sciences, 7(7): 35-42, (2013).

[9] Oluwafemi I. B. and Femi-Jemilohun O. J. "Propagation Profile and Signal Strength Variation of VHF Signal in Ekiti State Nigeria." I. J. Wireless and Microwave Technologies (IJWMT) DOI: 10.5815/ijwmt.2017.03.02, 3, 9-24 (2017).

[10] European Radiocommunications Committee Report 77 Field Strength Measurements along Route. Naples, Japan, (2000).

[11] Krebs P., Stocker M., Pezzatti G. B. and Conedera M. "An Alternative Approach to Transverse and Profile Terrain Curvature" International Journal of Geographic Information Science 1 - 24, (2015).

[12] Full User Manual Surfer 11Contouring and 3D Surface Mapping for Scientists and Engineers, pp 128, (2012).

\section{Author's Profile}

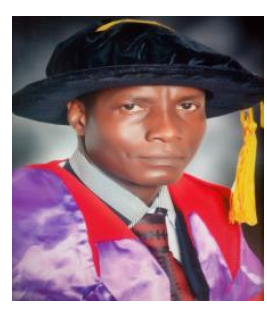

Dr. Adenodi Raphael Adewale is a lecturer in the Department of Physics, Adeyemi College of Education, Ondo, Nigeria. He holds a Bachelor of Science Degree in Physics Education, Master of Technology Degree in Electronic Measurements and Instrumentation as well as Doctor of Philosophy (Ph.D) in Communications Physics obtained from Obafemi Awolowo University, Ile-Ife, The Federal University of Technology, Akure and The Federal University of Technology, Akure, respectively. He has both teaching and research experiences for a period of thirteen years, led research team, published articles in some reputable journals, attended and presented research works in conferences. He is a member of International Union of Radio Science -Nigeria (URSI-NG), Science Association of Nigeria (SAN) and Teacher Registration Council of Nigeria (TRCN). 
How to cite this paper: Adenodi Raphael Adewale, "Investigating the Relationship between Coverage Area and Propagation Profile", International Journal of Wireless and Microwave Technologies(IJWMT), Vol.9, No.2, pp. 11-19, 2019.DOI: 10.5815/ijwmt.2019.02.02 